\title{
Full bulk spin polarization and intrinsic tunnel barriers at the surface of layered manganites
}

\author{
J. W. FREELAND ${ }^{1 *}$, K. E. GRAY², L. OZYUZER ${ }^{2 \dagger}$, P. BERGHUIS², ELVIRA BADICA², J. KAVICH', \\ H. ZHENG ${ }^{2}$ AND J. F. MITCHELL ${ }^{2}$
}

${ }^{1}$ Advanced Photon Source, ${ }^{2}$ Materials Science Division, Argonne National Laboratory, Argonne, Illinois 60439, USA

tPermanent address: Department of Phyisics, Izmir Institute of Technology, Izmir, TR-35437, Turkey

*e-mail: freeland@anl.gov

Published online: 12 December 2004; doi: 10.1038/nmat1280

Transmission of information using the spin of the electron as well as its charge requires a high degree of spin polarization at surfaces. However, at surfaces this degree of polarization can be quenched by competing interactions. Using a combination of surface-sensitive X-ray and tunnelling probes, we show for the quasi-twodimensional bilayer manganites that only the outermost $\mathrm{Mn}-\mathrm{O}$ bilayer is affected: it is a 1-nm-thick insulator that exhibits no long-range ferromagnetic order, whereas the next bilayer displays the full spin polarization of the bulk. Such an abrupt localization of the surface effects is due to the two-dimensional nature of the layered manganite, and the loss of ferromagnetism is attributed to weakened double exchange in the reconstructed surface bilayer and a resultant antiferromagnetic phase.

The creation of a well-defined surface insulator atop a fully spin-polarized bulk demonstrates the ability of two of the most demanding components of an ideal magnetic tunnel junction to self-assemble naturally.
1 igh spin polarization at surfaces and interfaces is a key component for transport of information using the spin degree of freedom of the electron ${ }^{1}$. Unfortunately, the physics and chemistry of surfaces can reduce this spin polarization through chemical inhomogeneity ${ }^{2,3}$, strain or surface reconstruction. Many half-metallic ferromagnetic oxides exhibit very high bulk magnetization, but the spin polarization at surfaces and interfaces declines more rapidly than the bulk magnetization as the Curie temperature, $T_{\mathrm{C}}$, is approached $\mathrm{d}^{4}$. Magnetic tunnel junctions ${ }^{5}$ as well as other probes of surface polarization ${ }^{6-10}$ in perovskite-based manganites indicate the presence of a non-ferromagnetic surface region, often called a 'dead layer,' of thickness $\sim 5 \mathrm{~nm}$.

We show for the quasi-two-dimensional bilayer manganites that the outermost $\mathrm{Mn}-\mathrm{O}$ bilayer, alone, is affected: this 1-nm-thick intrinsic nanoskin is an insulator with no long-range ferromagnetic order while the next bilayer displays the full spin polarization of the bulk. This unexpectedly abrupt termination is probably due to the reduced dimensionality of the crystal structure. That is, the electronic and magnetic coupling between the bilayers is markedly weaker than the corresponding intrabilayer couplings or the isotropic couplings found in non-layered ferromagnetic oxides.

A series of $\mathrm{La}_{2-2 x} \mathrm{Sr}_{1+2 x} \mathrm{Mn}_{2} \mathrm{O}_{7}$ single crystals were prepared by the floating zone method ${ }^{11}$ between $x=0.36$ and $x=0.5$. Sample preparation involved cleaving crystals in air under ambient conditions just before they were mounted in the ultra-high-vacuum measurement chamber. The natural cleavage planes between the bilayers (see Fig. 1) provide a clear advantage, and both atomic force microscopy and soft-X-ray rocking curves showed large, flat terraces. Surface-sensitive absorption measurements compare well with bulksensitive measurements, indicating that the surface is not degraded. These experiments involve data from several subsequent runs with different crystal batches that all demonstrate a reproducible nonferromagnetic surface layer. In addition, their bulk properties have been studied extensively by X-ray and neutron scattering, transport and magnetization ${ }^{12}$. In particular, these are double-exchange ${ }^{13,14}$ ferromagnets that exhibit symbiotic ferromagnetism and metallic conductivity below a $T_{\mathrm{C}}$ of $\sim 120-130 \mathrm{~K}$. Here we use polarized X-ray 


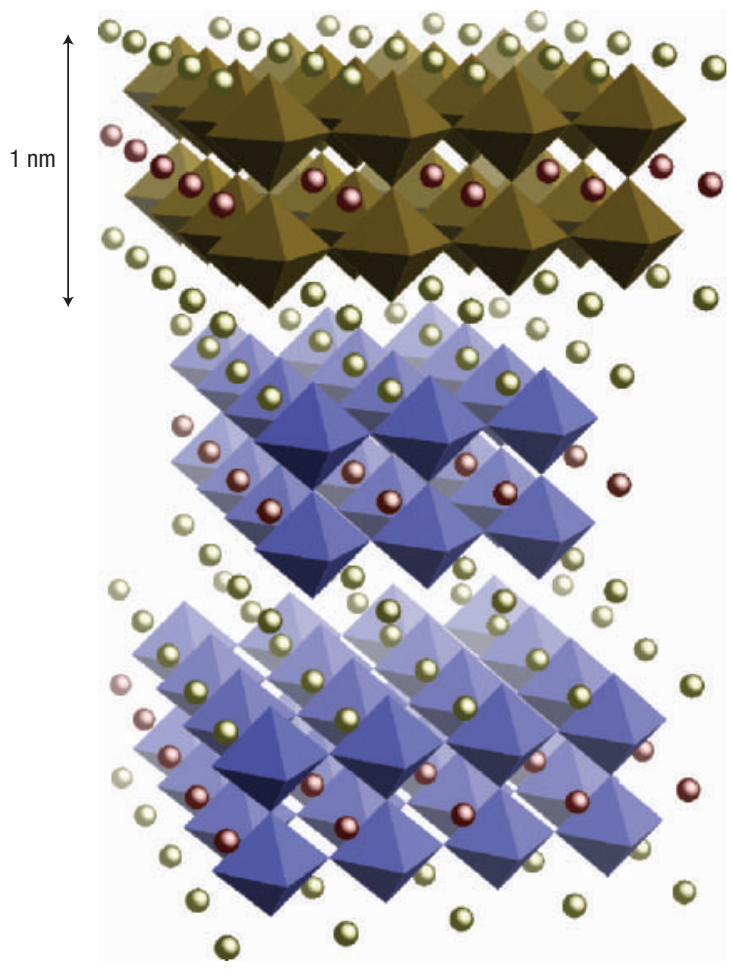

Figure 1 Structure of the naturally bilayered manganite $\mathrm{La}_{2-2 x} \mathrm{Sr}_{1+2 x} \mathrm{Mn}_{2} \mathrm{O}_{7}$. The $\mathrm{MnO}_{6}$ octahedra in the crystalline bulk are denoted in blue and ( $\mathrm{La}, \mathrm{Sr}$ ) sites are shown as yellow- and red-shaded spheres. The bilayer repeat distance is $\sim 10 \AA$. The non-ferromagnetic surface bilayer is coloured brown.

absorption and scattering (see Methods) at beamline $e^{15} 4$-ID-C of the Advanced Photon Source to study magnetism in the surface layers, and Au point-contact data and vacuum-gap tunnelling are used to assess the metallic or insulating nature of the surface.

X-ray resonant magnetic scattering ${ }^{16,17}$ (XRMS) allows us to map directly the magnetization profile near the surface by subtracting data for the X-ray helicity parallel $\left(I^{+}\right)$or antiparallel $\left(I^{-}\right)$to the magnetic moment (see Fig. 2). We tuned to the Mn L edge and the magnetic moment was aligned parallel to the crystal surface and the scattering plane with an applied magnetic field of 500 Oe, which was determined by field-dependent XRMS to be sufficient to achieve magnetic saturation. Additional magnetic information comes from simultaneous measurements of the polarization-dependent absorption, and we refer to the differences $\left(I^{+}-I^{-}\right)$in absorption as X-ray magnetic circular dichroism ${ }^{18}$ (XMCD), which is also shown in Fig. 2.

A change in the sign of the XRMS data with incident angle is clearly visible in Fig. 3a, and we interpret this as an interference of $\mathrm{X}$-rays scattering from the non-ferromagnetic surface layer. For a magnetic profile extending uniformly to the crystal surface, the sign of the XRMS is strictly independent of the scattering angle and is determined by the sign of the absorption XMCD. Crystals with a non-ferromagnetic surface layer will scatter strongly from both the chemical interface (cleaved surface) and the magnetic interface that is deeper down, because the chemical and magnetic scattering factors from the Mn $3 d$ electrons are of similar magnitudes ${ }^{17}$. Interference between these two distinct interfaces is readily observable in the XRMS signal. The data of Fig. 3a therefore prove unequivocally that the chemical and magnetic interfaces are out of registry in the layered manganite.
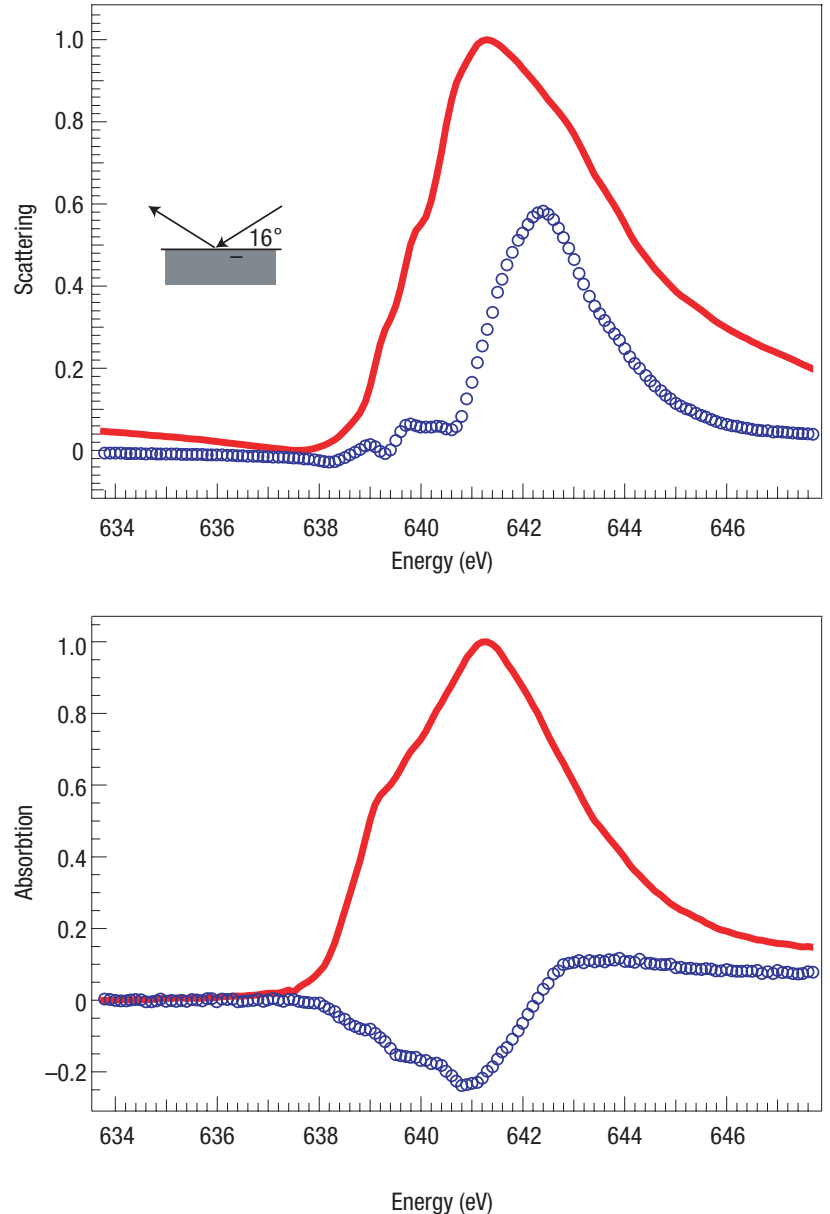

Figure 2 Polarization-dependent scattering and absorption data at the $M n L_{3}$ edge. The sum (red lines) and difference (blue circles) of signals at $35 \mathrm{~K}$ for the $X$-ray helicity parallel $\left(I^{+}\right)$or antiparallel $(\digamma)$ to the magnetic moment for the $x=0.36$ sample are shown. The XRMS is extremely sensitive to the magnetization profile (upper panel), whereas the magnetic properties averaged over the near-surface region are probed by XMCD (lower panel). Electronic properties are measured by $r+r$.

The details of the magnetic profile are determined by modelling the scattering data. Because X-rays at the Mn L edge have a wavelength of $\sim 2 \mathrm{~nm}$, the scattering from the structure is well described by the magneto-optics formalism ${ }^{19,20}$. By assembling a layered structure matching the unit cell, we used the dielectric tensor, determined from the polarization-dependent absorption, to model the scattering as a function of the magnetic profile near the surface. The case of a non-ferromagnetic surface bilayer provides the only match to the angle-dependent XRMS (solid lines in Fig. 3a). A comparison with simulations of magnetic profiles ranging from zero to two nonmagnetic bilayers (see Fig. 3b) demonstrates clearly that a single bilayer alone is non-ferromagnetic. Calculations show that as the second bilayer magnetization decreases, the XRMS peak position shifts continuously between the two positive peaks shown in Fig. $3 \mathrm{~b}$. From this we estimate that the spin polarization in the second bilayer at $35 \mathrm{~K}$ is the same as the bulk value, with an uncertainty of less than 20\%. The XRMS data thus reveal the presence of an extremely thin non-magnetic 'nanoskin,' below which subsequent bilayers are fully magnetized. 
a

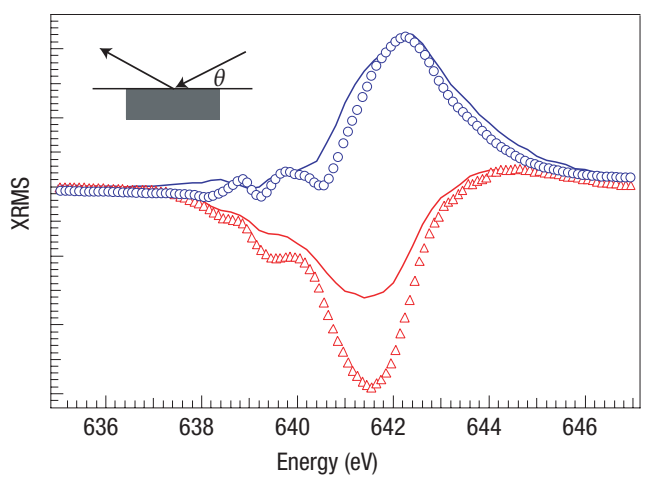

b

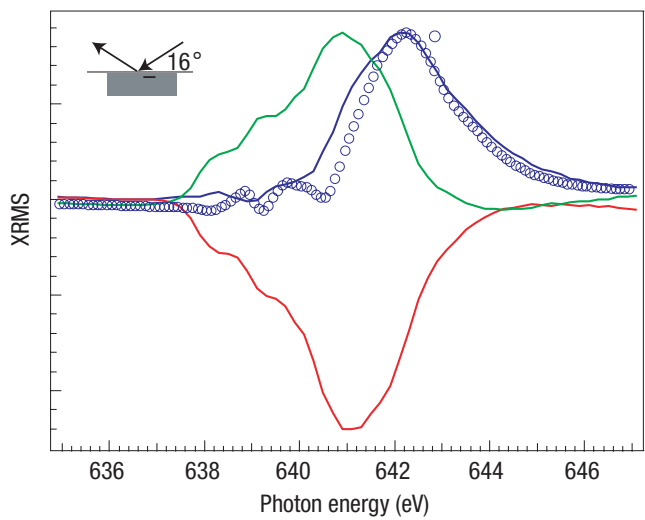

G

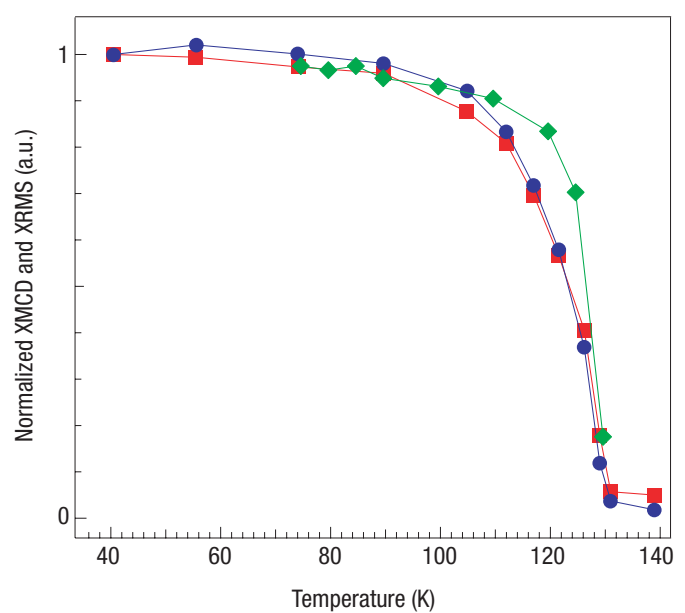

Figure 3 Magnetic profile determination and temperature dependence of the bulk-like sub-surface bilayer. a, The observed sign change (symbols) of our XRMS data at two angles (red, $5^{\circ}$; blue, $16^{\circ}$ ) can be simulated (lines) only by chemical and magnetic interfaces that are out of register. Data were taken at $35 \mathrm{~K}$. b. Calculated XRMS energy dependence at $\theta=16^{\circ}$ for the case of zero (red line), one (blue line) and two (green line) non-ferromagnetic bilayers. From the profound change in line shape we can pinpoint that the data (circles) are consistent only with the case of a single non-ferromagnetic bilayer. c, Normalized temperature-dependent magnetization of the second bilayer as measured by the XRMS (blue circles) and near-surface probe XMCD (red squares). These are virtually identical and show slight differences, only very close to $T_{\mathrm{C}}$, from the bulk magnetization from neutron scattering ${ }^{23}$ (green diamonds).
The near-surface XMCD data of Fig. 2, measured by electron yield, is about half the size of previous data on a three-dimensional perovskite manganite ${ }^{6}$ at low temperature. This finding is consistent with a non-ferromagnetic surface bilayer because the ratio is averaged over the escape of electrons with a mean free path of $\sim 1.5 \mathrm{~nm}$. We estimate that the integrated intensity from the top $1 \mathrm{~nm}$ (bilayer) corresponds to $\sim 50 \%$ of the total signal, so this ratio also requires nearly full bulk magnetization in the second bilayer.

In this field geometry, we are predominately sensitive to inplane moments, so if the spins in the outermost bilayer exhibited ferromagnetic order that was perpendicular to the layers, it would not be readily visible in the above data. However, we dismiss this for the following reasons. First, additional XMCD data were obtained in fields up to 7 T. For perpendicular field we found no net remanent moment. For parallel fields, XMCD shows a low-field saturation of magnetization in the second bilayer plus a linear component from the surface bilayer, which reaches $\sim 20 \%$ of saturation at $7 \mathrm{~T}$. If we assume that this increase is due to perpendicular ferromagnetic order in the surface bilayer, the increase would imply a huge uniaxial anisotropy of more than $10^{8} \mathrm{erg} \mathrm{cm}^{-3}$. That is near the upper limit of known materials that contain $4 f$ electrons and significant orbital moment. The layered manganites contain $3 d$ electrons and their orbital moment is quenched, resulting in typical ${ }^{21}$ anisotropy of less than $10^{6} \mathrm{erg} \mathrm{cm}^{-3}$. In addition, the surface bilayer is shown below to be an insulator: this precludes ferromagnetic double exchange, and insulating layered manganites are antiferromagnetic. It therefore seems reasonable that the enhanced surface magnetization at $7 \mathrm{~T}$ results from overcoming the antiferromagnetic superexchange energy (typically $\sim 10^{8} \mathrm{erg} \mathrm{cm}^{-3}$ in these materials ${ }^{22}$ ) in the outermost bilayer. To confirm this idea we measured a layered manganite with $x=0.5$, which is an A-type antiferromagnet below $200 \mathrm{~K}$ that is insulating in the bulk ${ }^{12}$. Here the A-type structure consists of two sheets of ferromagnetic spins within the bilayer that are oriented antiferromagnetically. Although there is no net moment at zero field for $x=0.5$, a magnetic field cants the ferromagnetic sheets to produce a moment, and the resulting magnetization measured by a magnetometer and XMCD is linear in field and roughly equal (to within 10\%). The magnetization increase for $x=0.5$ at $7 \mathrm{~T}$ is close to the $\sim 20 \%$ found above in the surface bilayer for $x=0.36$. From this we conclude that the surface bilayer for $x=0.36$ exhibits antiferromagnetic order, presumably because the double exchange is preferentially weakened by the surface reconstruction.

We now address the temperature dependence of the spin polarization in the second bilayer. All other half-metallic oxides ${ }^{4}$ show a significant loss of surface spin polarization as $T_{\mathrm{C}}$ is approached. For example, in the $\mathrm{La}_{1-x} \mathrm{Sr}_{x} \mathrm{MnO}_{3}$ system, the 'near surface' shows significant degradation from the bulk magnetization ${ }^{6}$ well below $T_{\mathrm{C}}$ and the 'very surface' displays a nearly linear decrease ${ }^{7}$ for all $T$ up to $T_{\mathrm{C}}$. Such a strong temperature-dependent degradation is also implied at interfaces in $\mathrm{La}_{1-x} \mathrm{Sr}_{x} \mathrm{MnO}_{3}$ magnetic tunnel junctions ${ }^{5}$, which show a complete loss of magnetoresistance for $T$ less than $\sim 0.7 T_{\mathrm{C}}$. In stark contrast, Fig. $3 \mathrm{c}$ compares the temperature dependence of the ratios $\left(I^{+}-I^{-}\right) /\left(I^{+}+I^{-}\right)$for XMCD and XRMS with the bulk magnetization ${ }^{23}$ determined from neutron scattering. For this sample $(x=0.36)$ the second bilayer retains the bulk value of the magnetization to within $\sim 10 \mathrm{~K}$ of $T_{\mathrm{C}}$. The only difference for $x=0.4$ was that the deviation from the bulk began $\sim 30 \mathrm{~K}$ below $T_{\mathrm{C}}$.

Now we address the question of whether the non-ferromagnetic surface bilayer is metallic. The electronic properties of the $c$-axis faces of these single crystals were probed with both scanning tunnelling spectroscopy (STS) and gold-tip point contacts on crystals that were cleaved in air at room temperature. The vacuum gap in STS guarantees tunnelling, whereas the gold point contacts can exhibit tunnelling only if an insulating barrier is present on the surface of the crystal. The data of Fig. 4 include numerous high-resistance Au 
point contacts taken sequentially at $4.2 \mathrm{~K}$ on crystals with $x=0.36$ plus representative STS data taken well above $T_{\mathrm{C}}$. They all exhibit a small degree of asymmetry, with the larger current for the crystal positive. This sequence at $4.2 \mathrm{~K}$ demonstrates the ability of the $\mathrm{Au}$ tip to clean the surface of contamination (presumably physisorbed). The initial 'gentle' touches (open circles and squares) yield featureless curves of $\log$ (current) against voltage, like the high-temperature data (inverted triangles) using STS that cannot clean the surface. Harder contacts significantly deform the Au tip, and in doing so progressively scrub the surface clean. The open-diamond data were taken after two hard contacts and the three solid-symbol data sets show the limiting characteristic after about ten hard contacts (note that the solid squares are taken on a different $x=0.36$ crystal surface after many hard contacts). The three solid-symbol data sets are also shown in the linear plot of the inset of Fig. 4.

These three sets of solid data points fit tolerably well, over a range of 10,000-fold in current, to the calculation of tunnelling (thick lines) through a square barrier of height $375 \mathrm{meV}$ using widths of $1.4 \mathrm{~nm}$ (solid squares) and $1.5 \mathrm{~nm}$ (solid circles and diamonds). We note that bandgaps of $\sim 300 \mathrm{meV}$ have been observed ${ }^{24}$ by STS in both the high-temperature paramagnetic and the lowtemperature charge-ordered insulating states of the non-layered manganite, $\mathrm{Bi}_{1-x} \mathrm{Ca}_{x} \mathrm{MnO}_{3}$. We also note that the exposed surface after cleaving the layered manganite crystals should be at the weakly bonded symmetry plane between the bilayers ${ }^{12}$. Thus, if the surface bilayer is insulating, the tunnelling distance from the topmost $\mathrm{Mn}-\mathrm{O}$ layer of the second bilayer to the Au point contact would be $\sim 1.4 \mathrm{~nm}$ in the absence of surface reconstruction in the insulating surface bilayer. We also point out that the open-diamond data in Fig. 4 are reproduced by summing parallel contributions from, first, the featureless (surface-contaminated) data of the open squares of Fig. 4 and, second, the tunnelling calculation that fits the solidsymbol data. This last observation is consistent with an incompletely cleaned surface after two 'hard' contacts. The distinct, reproducible barrier feature seen in the solid-symbol data of Fig. 4 attests to a high degree of barrier uniformity that may only be possible with an intrinsic barrier. Variations in barrier height, due to surface contamination for example, would spread out the feature in energy (that is, voltage), but the quality of the fit dismisses such variations. Similarly, the highly reproducible current-step size, from below to above $375 \mathrm{mV}$, rules out extrinsic variations in barrier width that would affect the current-step size exponentially.

Unfortunately, obtaining and/or maintaining a clean surface is far more difficult at higher temperatures, where the benefits of cryopumping are compromised. Even after repeated hard contacts, data taken at $77 \mathrm{~K}$ showed only occasionally a hint of the weak structure seen in the open-diamond data of Fig. 4. At temperatures above $T_{\mathrm{C}}$, all data were featureless, emulating that of the contaminated surfaces taken after 'gentle' touches at $4.2 \mathrm{~K}$. This similarity might imply that the density-of-states for tunnelling (through the combination of contamination layer and insulating surface bilayer) is insensitive to whether the bulk manganite beneath is a bad metal (below $T_{\mathrm{C}}$ ) or a bad insulator (above $T_{\mathrm{C}}$ ). One should recognize that in high fields the conductivity by thermal activation above $T_{\mathrm{C}}$ approaches the low-temperature metallic conductivity (see Fig. 7 of ref. 25), with its short mean free path ${ }^{26}$ of $\sim 1.5 \mathrm{~nm}$. This comparable conductivity for the insulator results from a significant lowering of the thermal activation barrier because of double exchange when high fields align the spins ${ }^{27}$. Because the double-exchange activation barrier is completely absent for tunnelling to a non-magnetic counterelectrode such as $\mathrm{Au}$, we can expect little effect except for the possible appearance of a gap at the Fermi level. The present data do not consistently distinguish such a gap, but they do not rule it out.

Because the crossover from the metallic state at $4.2 \mathrm{~K}$ to the insulating state above $T_{\mathrm{C}}$ was inaccessible, point-contact tunnelling

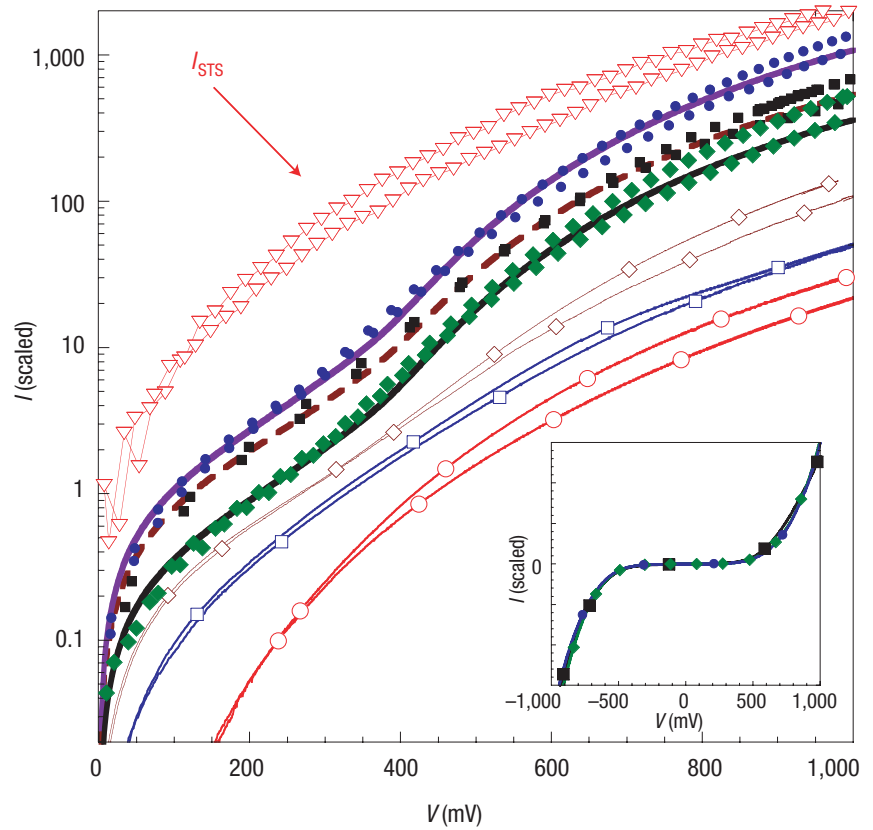

Figure 4 The log current-voltage characteristics of numerous high-resistance Au point-contacts (PT) taken at $4.2 \mathrm{~K}$ on a crystal $x=0.36$, plotted together with representative STS data taken well above $T_{\mathrm{c}}$. The curves have been displaced for clarity of presentation; the unknown junction areas scale the actual currents. Five of the PT curves were part of a sequence, and they demonstrate the surface-cleaning ability of the malleable Au tip when it is deformed by hard contact. The results shown by open circles and squares were taken during initial 'soft' contacts. The open diamond data were taken after two 'hard' contacts and the limiting behaviour of the solid diamonds and circles were taken after about ten 'hard' contacts. The solid squares represent the limiting case for another cleaved surface on a crystal with $x=0.36$. The thick lines are fits to the standard tunnelling model for the three sets of solid-symbol data, using a barrier height of $375 \mathrm{meV}$ and a width of $1.4-1.5 \mathrm{~nm}$. The inability of the scanning tunnelling microscope tip to clean the surface led to the featureless STS curve that was similar to the PT data in the limit of only non-cleaning 'soft' touches. Inset, solid-symbol data on a linear scale.

data were collected on an insulating layered manganite crystal with $x=0.48$, which exhibits an insulator-metal transition ${ }^{25}$ in fields of 3-4 T (Fig. 5b, c) at $4.2 \mathrm{~K}$. The featureless, zero-field tunnelling curve shown in Fig. 5a was repeatable even after the requisite number of hard contacts to clean the surface. In zero field, the high resistance of the insulating crystal also contributes to the point-contact voltage. Upon increasing the field to $6 \mathrm{~T}$, the crystal resistance decreases by more than four orders of magnitude ${ }^{25}$ and the characteristic shoulder seen in the solid data symbols of Fig. 4 appeared, which shows the presence of an insulating surface bilayer. The known magneto-thermal hysteresis in this material ${ }^{25}$ is reproduced in the tunnelling - the shoulder did not disappear on lowering the field to zero at $4.2 \mathrm{~K}$, but it disappeared after briefly cycling the crystal in zero field to $95 \mathrm{~K}$ and back to $4.2 \mathrm{~K}$.

Thus we conclude that, at least at low temperatures, the topmost bilayer is an insulator with a $375-\mathrm{meV}$ bandgap. The insulating and non-ferromagnetic regions therefore coincide. The simultaneous absence of magnetic order and metallic conductivity in the top bilayer is entirely consistent with the double-exchange mechanism ${ }^{13,14}$ used to explain conductivity and ferromagnetism below $T_{\mathrm{C}}$ in manganites. The abrupt changes in only the topmost bilayer are probably due to the weak electronic and magnetic coupling between bilayers 

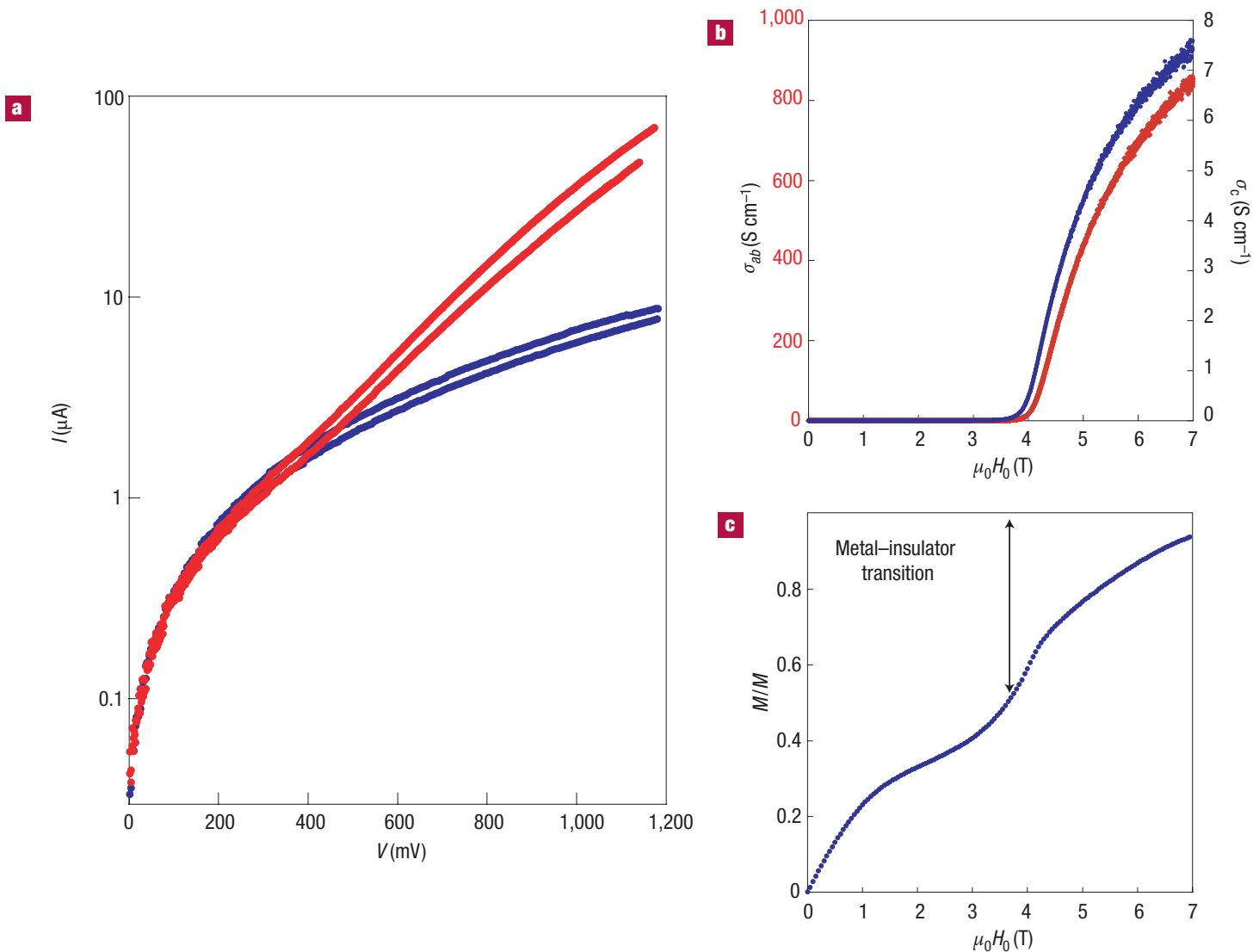

Figure $\mathbf{5}$ Field-induced metal-insulator transition for a layered manganite with $\boldsymbol{x}=\mathbf{0 . 4 8}$. a, Gold point-contact data showing how the featureless zero-field pointcontact curve (blue) switches in $6 \mathrm{~T}$ to one (red) showing a barrier, similar to that seen in the solid data of Fig. 4 , on a metallic bulk manganite crystal. This composition exhibits an insulator-metal transition at $4.2 \mathrm{~K}$ in a magnetic field of 3-4 T, as seen in $\mathbf{b}$ for both the $c$-axis (blue) and ab-plane (red) conductivity for $x=0.48$. These conductivity data are taken from ref. 25 on a crystal showing very similar magnetization data to that in $\mathbf{c}$, the crystal used for the tunnelling of $\mathbf{a}$.

engendered by the crystal structure ${ }^{12}$ and not found in non-layered magnetic oxides.

Because of the complex interplay of the structural, electronic, orbital and magnetic degrees of freedom there exist many possibilities for the formation of an insulating, non-ferromagnetic layer at the surface. Although our results do not directly pinpoint the origin of this surface layer, they can eliminate some possibilities. Surface roughness can be ruled out by our observation of large atomically flat terraces created on cleaving the crystal. Significant deviations from stoichiometry at the surface due to Sr segregation, which is observed in the perovskite system ${ }^{3}$, or oxygen loss would result in a much larger change in the electronic structure at the surface (from the $I^{+}+I^{-}$data for both XRMS and XMCD) than that observed. If such disorder is ruled out, a prime candidate might be surface strain $^{28}$ due to surface reconstruction or a change in atomic coordination $^{29}$ that could stabilize orbital- or charge-ordered insulating states that, in turn, would suppress the ferromagnetic double-exchange mechanism.

In summary, we have shown that the surface bilayer of aircleaved layered manganites forms an antiferromagnetic insulating nanoskin composed of a single bilayer unit, and the next bilayer is metallic and retains the full bulk spin polarization up to nearly $T_{\mathrm{C}}$. Although the surface is prepared in air under ambient conditions, indications are that because no strong bonds are broken during the cleavage process, the surface is stable and the results represent an intrinsic property. An important aspect of this result is that the outermost bilayer could act as an intrinsic tunnel barrier between the fully spin-polarized bilayer beneath and a subsequently deposited ferromagnetic counterelectrode. Artificially grown oxide heterostructures are typically plagued by inhomogeneities in the barrier that can degrade performance. In contrast, for layered manganite crystals, nature provides this tunnel barrier with uniform thickness and properties.

\section{METHODS}

The resonant absorption and scattering processes operate by tuning the X-ray energy to a threshold corresponding to excitation from a $2 p$ core level to the unoccupied $3 d$ states of Mn. Because this energy is specific to $\mathrm{Mn}$, it provides an element-selective way to study the electronic structure of $\mathrm{Mn}$. If we add polarization dependence then magnetic information can be extracted as well. In practice this involves measurements of the signals with the sample magnetization parallel $\left(I^{+}\right)$or anti-parallel $\left(I^{-}\right)$to the incident beam polarization. With the circularly polarized beam incident at grazing angles of $5-16^{\circ}$, a constant magnetic field of \pm 500 Oe was then applied in the plane of the surface at each point of the energy scan to achieve this configuration. Field-dependent measurements performed in situ show that $500 \mathrm{Oe}$ is sufficient to achieve magnetic saturation for all the samples. The sum $\left(I^{+}+I^{-}\right)$consists of purely electronic information, whereas the difference $\left(I^{+}-I^{-}\right)$is magnetic in origin. For the case of absorption this signal is purely magnetic, but in the case of scattering it consists of a charge-magnetic interference term. It is this term that provides the contrast necessary to extract the magnetic profile.

For junctions with low specific resistance, the spreading resistance in the fairly resistive manganite 'substrate' contributes to the measured voltage. Spectral features of such point contacts exhibit a dependence on magnetic field in excellent agreement with the resistivity of the layered manganite crystal measured in a separate experiment by the methods discussed in refs 25 and 26 . We therefore used only data from our highest-resistance Au point-contact junctions in Fig. 4, although lower-resistance junctions were made during the 'hard' contacts used for cleaning the manganite surface.

Received 25 May 2004; accepted 22 September 2004; published 12 December 2004. 


\section{References}

1. Wolf, S. A. et al. Spintronics: A spin-based electronics vision for the future. Science 294, 1488-1495 (2001).

2. Borca, C. N. et al. Electronic structure modifications induced by surface segregation in $\mathrm{La}_{0.65} \mathrm{~Pb}_{0.35} \mathrm{MnO}_{3}$ thin films. Europhys. Lett. 56, 722-728 (2001).

3. Dulli, H., Dowben, P. A., Liou, S.-H. \& Plummer, E. W. Surface segregation and restructuring of colossal-magnetoresistive manganese perovskites $\mathrm{La}_{0.65} \mathrm{Sr}_{0.35} \mathrm{MnO}_{3}$. Phys. Rev. B 62, R14629-R14632 (2000).

4. Coey, J. M. D. \& Chien, C. L. Half-metallic ferromagnetic oxides. Mater. Res. Soc. Bull. 28, 720-724 (2003).

5. O'Donnell, J., Andrus, A. E., Oh, S., Colla, E. V. \& Eckstein, J. N. Colossal magnetoresistance magnetic tunnel junctions grown by molecular beam epitaxy. Appl. Phys. Lett. 76, 1914-1916 (2000).

6. Park, J. H. et al. Magnetic properties at the surface boundary of a half-metallic ferromagnetic $\mathrm{La}_{0.7} \mathrm{Sr}_{0.3} \mathrm{MnO}_{3}$. Phys. Rev. Lett. 81, 1953-1956 (1998).

7. Park, J. H. et al. Direct evidence for a half-metallic ferromagnet. Nature 392, 794-796 (1998).

8. Soulen R. J. Jr. et al. Measuring the spin polarization of a metal with a superconducting point contact. Science 282, 85-88 (1998).

9. Ott, F. et al. Interface magnetism of $\mathrm{La}_{0.7} \mathrm{Sr}_{0.3} \mathrm{MnO}_{3}$ probed by neutron reflectometry. J. Magn. Magn. Mater. 211, 200-205 (2000).

10. Sun, J. Z. et al. Thickness dependent magneto-transport in ultrathin manganite films. Appl. Phys. Lett. 74, 3017-3019 (1999).

11. Mitchell, J. F. et al. Charge delocalization and structural response in layered $\mathrm{La}_{1.2} \mathrm{Sr}_{1.8} \mathrm{Mn}_{2} \mathrm{O}_{7}$ : Enhanced distortion in the metallic regime. Phys. Rev. B 55, 63-66 (1997).

12. Mitchell, J. F. et al. Spin, charge, and lattice states in layered magnetoresistive oxides. J. Phys. Chem. B 105, 10731-10745 (2001).

13. Zener, C. Interaction between the d-shells in the transition metals. II. Ferromagnetic compounds of manganese with perovskite structure. Phys. Rev. 82, 403-405 (1951).

14. Anderson, P. W. \& Hasagawa, H. Considerations on double exchange. Phys. Rev. 100, 675-68 (1955).

15. Freeland, J. W. et al. A unique polarized $\mathrm{x}$-ray facility at the advanced photon source. Rev. Sci. Instr. 73, 1408-1410 (2002).

16. Kao, C. et al. Magnetic-resonance exchange scattering at the iron $\mathrm{L}_{\mathrm{II}}$ and $\mathrm{L}_{\mathrm{III}}$ edges. Phys. Rev. Lett. 65 , 373-376 (1990).
17. Sacchi, M. \& Hague, C. F. Magnetic coupling in thin layers and superlattices investigated by resonant scattering of polarized soft x-rays. Surf. Rev. Lett. 9, 811-820 (2002).

18. Chen, C. T. et al. Experimental confirmation of the $\mathrm{x}$-ray magnetic circular dichroism sum rules for Fe and Co. Phys. Rev. Lett. 75, 152-155 (1995).

19. Kortright, J. B. \& Kim, S.-K. Resonant magneto-optical properties of Fe near its $2 p$ levels: Measurement and applications. Phys. Rev. B 62, 12216-12228 (2000).

20. Zak, J., Moog, E. R., Liu, C. \& Bader, S. D. Magneto-optics of multilayers with arbitrary magnetization directions. Phys. Rev. B 43, 6423-6429 (1991).

21. Welp, U. et al. Magnetic anisotropy and domain structure of the layered manganite $\mathrm{La}_{1.36} \mathrm{Sr}_{1.64} \mathrm{Mn}_{2} \mathrm{O}_{7}$. Phys. Rev. B 62, 8615-8618 (2000).

22. Perring, T. G. et al. Spectacular doping dependence of interlayer exchange and other results on spin waves in bilayer manganites. Phys. Rev. Lett. 87, 217201-1-217201-4 (2001).

23. Osborn, R. et al. Neutron scattering investigation of magnetic bilayer correlations in $\mathrm{La}_{1.2} \mathrm{Sr}_{1.8} \mathrm{Mn}_{2} \mathrm{O}_{7}$ : Evidence of canting above T. Phys. Rev. Lett. 81, 3964-3967 (1998).

24. Renner, Ch., Aeppli, G., Kim, B.-G., Soh, Y.-A. \& Cheong, S.-W. Atomic-scale images of charge ordering in mixed-valence manganite. Nature 416, 518-521 (2002).

25. Li, Qing'an, Gray, K. E., Berger, A \& Mitchell, J. F. Electronically driven first-order metal-insulator transition in layered manganite $\mathrm{La}_{1.04} \mathrm{Sr}_{1.96} \mathrm{Mn}_{2} \mathrm{O}_{7}$ single crystals. Phys. Rev. B 67, 184426 (2003).

26. Li, Qing'an, Gray, K. E. \& Mitchell, J. F. Metallic conductance below $T_{\mathrm{C}}$ inferred by quantum interference effects in layered $\mathrm{La}_{1.2} \mathrm{Sr}_{1.8} \mathrm{Mn}_{2} \mathrm{O}_{7}$ single crystals. Phys. Rev. B 63, 024417 (2000).

27. Dionne, G. F. Magnetic exchange and charge transfer in mixed-valence manganites and cuprates. J. Appl. Phys. 79, 5172-5174 (1996).

28. Calderon, M. J., Millis, A. J. \& Ahn, K. H. Strain selection of charge and orbital ordering patterns in half-doped manganites. Phys. Rev. B 68, R100401 (2003).

29. Filippetti, A. \& Pickett, W. E. Double exchange driven spin pairing at the (001) surface of manganites. Phys. Rev. B 62, 11751-11755 (2000).

\section{Acknowledgements}

The research, including the use of the Advanced Photon Source, was supported by the US Department of Energy (DOE), Basic Energy Sciences, under contract W-31-109-ENG-38. Correspondence and requests for materials should be addressed to J.W.F.

\section{Competing financial interests}

The authors declare that they have no competing financial interests. 